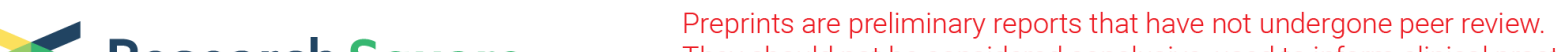 Research Square They should not be considered conclusive, used to inform clinical practice, or referenced by the media as validated information.
}

\section{Patient Reported Outcomes and Complications during Head \& Neck Cancer Radiotherapy before versus during the COVID-19 Pandemic}

Sarah Hamilton ( $\sim$ SHamilton7@bccancer.bc.ca)

BC Cancer Agency Vancouver Centre https://orcid.org/0000-0002-1816-2599

Nicole Chau

BC Cancer Agency: British Columbia Cancer Agency

Eric Berthelet

BC Cancer Agency: British Columbia Cancer Agency

Jonn Wu

BC Cancer Agency: British Columbia Cancer Agency

\section{Eric Tran}

BC Cancer Agency: British Columbia Cancer Agency

Melanie Chevrier

BC Cancer Agency: British Columbia Cancer Agency

Victoria Lau

BC Cancer Agency: British Columbia Cancer Agency

Matthew Chan

BC Cancer Agency: British Columbia Cancer Agency

Kimberly DeVries

BC Cancer Agency: British Columbia Cancer Agency

Vincent Lapointe

BC Cancer Agency: British Columbia Cancer Agency

Robert A Olson

BC Cancer Agency: British Columbia Cancer Agency

\section{Research Article}

Keywords: head and neck cancer, radiotherapy, COVID-19, patient reported outcomes, treatment toxicity

Posted Date: October 21st, 2021

DOI: https://doi.org/10.21203/rs.3.rs-905308/v1 
License: (c) (i) This work is licensed under a Creative Commons Attribution 4.0 International License. Read Full License

Version of Record: A version of this preprint was published at Supportive Care in Cancer on November 26th, 2021. See the published version at https://doi.org/10.1007/s00520-021-06703-x. 


\section{Abstract \\ Purpose}

This study compares patient reported outcomes and treatment related complications during radiotherapy before (August 2019 -Feb 2020) versus during (March -October 2020) the COVID-19 pandemic.

\section{Materials and Methods}

The MD Anderson Symptom Inventory - Head \& Neck Module was used to assess curative intent H\&N cancer patients' symptoms during radiotherapy.

\section{Results}

There were 158 patients in the pre-pandemic cohort and 137 patients in the pandemic cohort. There was a trend towards more patients requiring an enteral feeding during the pandemic ( $21 \%$ vs $30 \%, p=0.07)$. Weight loss was higher during the pandemic (mean $-5.6 \%$ vs $6.8 \%, p=0.03$ ). On multivariate analysis, treatment during the pandemic was associated with higher symptom scores for coughing/choking while eating ( 2.7 vs $2.1, p=0.013)$.

\section{Conclusions}

Complication rates during $\mathrm{H} \& \mathrm{~N}$ radiotherapy during the COVID-19 pandemic were similar at our institution relative to the pre-pandemic era although weight loss was greater and patients reported more severe choking/coughing while eating.

\section{Introduction:}

Radiotherapy is commonly used for the treatment of head and neck (H\&N) cancer. Radical radiotherapy is associated with significant cumulative acute side effects including severe mucositis, pain, dysgeusia, dysphagia and weight loss.[1] The substantial toxicity from H\&N radiotherapy can have significant impacts on patients quality of life and is associated with a high risk of treatment related morbidity and mortality.[1]-[5]

The COVID-19 outbreak began in mid-March 2020 in Canada, leading to an unprecedented shut down of non-essential services in the province of British Columbia. However, BC Cancer continued to provide essential radiotherapy for patients with $\mathrm{H} \& \mathrm{~N}$ cancer per our standard of care and radiation oncologists continued to evaluate these patients in person during their radiation treatment. Despite this, several support services were not consistently available throughout the pandemic, such as volunteer driver support to attend appointments, in person patient and family counselling sessions, speech language 
pathology instrumental assessments and reduced cost housing for out of town patients. Additionally, many patients had reduced personal and family support available due to social distancing measures in place. Furthermore, access to in person visits with family physicians and specialists were limited. Some patients presented with more locally advanced disease, due to delays in diagnosis during the pandemic. [6][7]

Due to the above factors, it was our clinical perception that a higher proportion of our patients developed greater toxicities during the COVID-19 pandemic. Therefore, we aimed to compare patient reported outcomes (PROs) and complications during $\mathrm{H} \& \mathrm{~N}$ radical radiotherapy for patients undergoing treatment from March 2020-Sept 2020, relative to the preceding period from Aug 2019-Jan 2020. This was performed to assess if the rate of treatment related complications were higher and PROs were worse during the COVID-19 pandemic and to have a better understanding of the pandemic's impact on H\&N patients' quality of life during radiotherapy.

\section{Materials And Methods:}

The Prospective Outcomes and Support Initiative (POSI) collects PROs across all tumour sites at our multi-centre institution.[8] Patients undergoing radical radiotherapy for $\mathrm{H} \& \mathrm{~N}$ cancer to any sub-site complete the MD Anderson Symptom Inventory for head and neck cancer module (MDASI-HN) on the day of their radiation planning scan using an iPad as the baseline assessment.[9], [10][11] The MDASI-HN is a $\mathrm{H} \& \mathrm{~N}$ cancer specific questionnaire, that contains the core MDASI's 13 symptom and 6 interference questions with an additional 9 symptom questions relevant to $\mathrm{H} \& \mathrm{~N}$ cancer. Each symptom is scored on a scale of zero to ten, with ten being the most severe.[11] Each week during radiotherapy, patients are provided the iPad to repeat the MDASI-HN questionnaire (weekly surveys). For the purpose of this study, patients were analyzed based on two distinct time periods: those who both started and completed radiotherapy before the COVID-19 pandemic (pre-COVID cohort, August 2019 to Jan 2020) relative to those who started and completed radiotherapy during the COVID-19 pandemic (March 2020 to October 2020.) Patients who received palliative radiotherapy were excluded, as well as those patients who started pre-pandemic (Feb 2020) and completed radiotherapy during the pandemic (Mar 2020). Patients who did not complete a baseline questionnaire and at least one weekly questionnaire were excluded from the PRO analysis. Clinical, pathologic and treatment data was abstracted retrospectively. Variables abstracted included age at diagnosis, sex, tumour site, T-stage, $\mathrm{N}$-stage, smoking status, alcohol usage, Eastern Cooperative Oncology Group (ECOG) performance status, surgery, radiation dose and use of concurrent chemotherapy.

\section{Treatment Details}

Patients were treated with Volumetric Arc Therapy (VMAT) radiotherapy techniques to 70 Gray (Gy) in 35 fractions. Some patients received altered fractionation schedules including $55 \mathrm{~Gy}$ in 20 fractions (early larynx cancers only), $60 \mathrm{~Gy}$ in 25 fractions, $66 \mathrm{~Gy}$ in 30 fractions to gross disease. Post-operative patients received 60 to $66 \mathrm{~Gy}$ in 30 to 33 fractions. Concurrent chemotherapy was used for patients with locally 
advanced disease $(\mathrm{T} 3+, \mathrm{N}+)$ for definitive therapy and post-operatively for extra nodal extension or positive margins. Cisplatin weekly $(40 \mathrm{mg} / \mathrm{m} 2)$ or every 3 weeks $(100 \mathrm{mg} / \mathrm{m} 2)$ was used, or cetuximab ( $400 \mathrm{mg} / \mathrm{m} 2$ loading dose day $-7,250 \mathrm{mg} / \mathrm{m} 2$ weekly) for patients with contraindications to cisplatin. There was no changes to the above treatment recommendations (dose, fractionation, usage of concurrent chemotherapy) during the COVID-19 pandemic.

\section{Data Analysis}

Baseline patient, disease and treatment characteristics and complication rates were compared between the two cohorts using Chi-square test and independent sample t-test.

The 28 symptom scores of the MDASI-HN were analyzed across 7 weekly questionnaires completed during radiotherapy. Subscale score totals were calculated based on the arithmetic mean of items in the subscale: Core Symptoms - question 1-13, H\&N Module Symptoms - question 14-22 and Interference - question 23-28. When calculating subscale scores, a majority of the subscale's items must have been responded to (i.e., 7 of the 13 Core symptom severity items, 5 of the $9 \mathrm{H} \& \mathrm{~N}$ module symptom items, or 4 of the 6 Interference items would represent the majority of the items for the subscales). If the patient responded to fewer than half of the subscale's items at a given assessment, the subscale total for that weekly assessment was considered missing.[11] The mean average score for each symptom and subscale scores pre-COVID versus during COVID were compared using independent sample t-test with Bonferroni correction for multiple comparisons. Group differences were considered significant if $p<0.002$ for the 28 individual symptom items and $p<0.02$ for the 3 subscale score totals.

Linear mixed models were used to assess trends in symptom progression over time and test cohort group effects in symptom severity for each MDASI-HN individual symptom item and three overall symptom subscale scores. Mixed models were used due to the presence of repeated measures data, where data collected at one point in time is not independent of the data collected at another time in the study, and due to the capability of analyzing data with missing values. Univariate linear mixed models were fit with time and cohort as fixed effects and patients as random effects to examine the effect on mean symptom severity for each individual symptom question or subscale total as the outcome. Multivariate linear mixed models were used to evaluate correlates of symptom severity for the most prominent MDASI-HN symptom questions (i.e., those showing the highest symptom severity difference between cohort groups in univariate analysis). The three symptom outcomes analyzed were: Q12 - vomiting, Q16 choking/coughing while eating and Q21 - mouth/throat sores. Correlates of symptom severity were also analyzed for overall symptom subscale scores (Core, $\mathrm{H} \& \mathrm{~N}$, Interference) on multivariate analysis using linear mixed models. Multivariate linear mixed models were fit with time, cohort and covariates of interest as fixed effects and patients as random effects to examine the influence on mean symptom severity. Covariates were selected for inclusion based on clinical importance and statistical significance and included age at treatment, sex, smoking status, alcohol use, ECOG performance status, percent weight loss, T-stage, N-stage, tumor site, receipt of chemotherapy, surgery, and radiation dose. Interaction effects were also tested to determine if the effect of symptom severity over time varied between cohort pre/during COVID time periods. 
Statistical analysis was performed using SAS Version 9.4 (SAS Institute Inc., Cary, NC). P-values of $<0.05$ were considered statistically significant, unless otherwise noted when Bonferroni corrections were used, and all tests were two-tailed. The study was approved by our intuitions research ethics board.

\section{Results:}

\section{Patient Cohorts}

Table 1 shows the baseline characteristics of the pre-COVID and during COVID cohorts. Mean age, sex distribution, ECOG status and alcohol usage were similar between the cohorts $(p>0.05)$. There were more current smokers during the COVID pandemic (20\% vs $10 \%, p=0.03)$. T-stage, $\mathrm{N}$-stage and tumour site distribution were similar pre COVID and during COVID. More patients received concurrent chemotherapy during COVID ( $58 \%$ vs $42 \%, p=0.03)$ but radiation doses, and usage of surgery were similar between the cohorts. 
Table 1

Patient cohorts pre-COVID 19 pandemic and during COVID-19 pandemic.

\begin{tabular}{|c|c|c|c|}
\hline Characteristic & $\begin{array}{l}\text { Pre-COVID } \\
\text { Cohort } \\
\text { n (\%) }\end{array}$ & $\begin{array}{l}\text { During COVID } \\
\text { Cohort } \\
\mathrm{n}(\%)\end{array}$ & p-value \\
\hline \multicolumn{4}{|l|}{ Demographics } \\
\hline Mean Age (range) & $\begin{array}{l}63.8 \text { years } \\
(26-91 \text { years })\end{array}$ & $\begin{array}{l}63.2 \text { years } \\
(27-94 \text { years })\end{array}$ & 0.48 \\
\hline \multicolumn{4}{|l|}{ Sex } \\
\hline Male & $126(80 \%)$ & $105(77 \%)$ & 0.52 \\
\hline Female & $32(20 \%)$ & $42(23 \%)$ & \\
\hline \multicolumn{4}{|l|}{ Smoking Status } \\
\hline Lifelong non-smokers & $72(46 \%)$ & $56(41 \%)$ & \\
\hline Ex-smoker (quit $\geq 1$ year ago) & $71(45 \%)$ & $53(39 \%)$ & 0.03 \\
\hline Current smokers (quit < 1 year ago) & $15(10 \%)$ & $28(20 \%)$ & \\
\hline \multicolumn{4}{|l|}{ Alcohol Use } \\
\hline Yes & 37 (23\%) & 37 (27\%) & 0.50 \\
\hline No & $121(77 \%)$ & $100(73 \%)$ & \\
\hline \multicolumn{4}{|l|}{ ECOG } \\
\hline 0 & $51(32 \%)$ & $58(42 \%)$ & 0.27 \\
\hline 1 & $91(58 \%)$ & $65(47 \%)$ & \\
\hline 2 & $12(8 \%)$ & $9(7 \%)$ & \\
\hline 3 & $4(3 \%)$ & $5(4 \%)$ & \\
\hline \multicolumn{4}{|l|}{ Disease Factors } \\
\hline \multicolumn{4}{|l|}{ T Stage } \\
\hline T1/T2 & $90(57 \%)$ & $78(57 \%)$ & 0.99 \\
\hline T3/T4 & $68(43 \%)$ & $59(43 \%)$ & \\
\hline \multicolumn{4}{|l|}{ N Stage } \\
\hline Node Negative & $50(32 \%)$ & 34 (25\%) & 0.19 \\
\hline Node Positive & 108 (68\%) & 103 (75\%) & \\
\hline
\end{tabular}




\begin{tabular}{|llll|}
\hline Characteristic & $\begin{array}{l}\text { Pre-COVID } \\
\text { Cohort } \\
\text { n (\%) }\end{array}$ & $\begin{array}{l}\text { During COVID } \\
\text { Cohort } \\
\text { n (\%) }\end{array}$ & p-value \\
\hline Tumour Site & & & \\
\hline Oropharynx & $70(44 \%)$ & $65(47 \%)$ & 0.18 \\
\hline Larynx/hypopharynx & $29(19 \%)$ & $14(11 \%)$ & \\
\hline Nasopharynx/paranasal sinus & $23(14 \%)$ & $16(12 \%)$ & \\
\hline Oral cavity & $17(11 \%)$ & $21(15 \%)$ & \\
\hline Salivary gland & $7(4 \%)$ & $10(7 \%)$ & \\
\hline Thyroid & $6(4 \%)$ & $3(2 \%)$ & 0.34 \\
\hline Other/primary unknown & $6(4 \%)$ & $6(4 \%)$ & \\
\hline Treatment Factors & & & \\
\hline Concurrent Chemotherapy & & 50.03 & \\
\hline None & $92(58 \%)$ & $57(42 \%)$ & \\
\hline Cisplatin 100mg/m2 q3weekly & $21(13 \%)$ & $13(10 \%)$ & \\
\hline Cisplatin 40mg/m2 weekly & $44(28 \%)$ & $63(46 \%)$ & \\
\hline Cetuximab & $1(1 \%)$ & $4(3 \%)$ & \\
\hline Surgery & & & \\
\hline Yes & $59(37 \%)$ & $57(63 \%)$ & \\
\hline No & $66 \mathrm{~Gy}(50-70 \mathrm{~Gy})$ & \\
\hline Mean Total Dose (range) & & & \\
\hline & & & \\
\hline
\end{tabular}

\section{Complication Rate}

Complication rates were similar pre versus during COVID. There were more patients requiring enteral feeding during COVID (30\% vs $21 \%)$, but this did not reach statistical significance $(p=0.07)$. The mean percentage weight loss was higher during the pandemic at $6.8 \%$ loss compared to baseline versus $5.6 \%$ pre-pandemic $(p=0.03)$. Rates of aspiration pneumonia and hospitalization during radiotherapy were similar pre versus during COVID. No patients in our study developed documented COVID-19 infection during radiotherapy.

MDASI-HN compliance 
Of the 295 patients included in the study, 278 patients completed a baseline questionnaire and at least one weekly assessment during radiation; these patients were included for subsequent analysis. Four or more weekly questionnaires (of 7 ) were completed by $236 / 278$ patients ( $85 \%$ ). The average number of weekly questionnaires completed was 5 (range: 1-8).

\section{Symptom Severity}

Mean average symptom scores (across the duration of radiation treatment) were highest (maximum score $=10$ ) for tasting food (5.06), dry mouth (4.46), fatigue/tiredness (4.33), swallowing/chewing (4.31), lack of appetite (4.07), and pain (3.89). Similarly, mean maximum symptom scores (maximum symptom score at any time during radiation treatment) were highest for tasting food (6.80), swallowing/chewing (6.20), dry mouth (6.10), lack of appetite (5.97) fatigue/tiredness (5.88), and pain (5.77).

Mean average symptom scores and subscale scores comparing pre COVID versus during COVID scores are shown in Table 3.

Table 2

Complications pre COVID-19 pandemic versus during COVID-19 pandemic

\begin{tabular}{|llll|}
\hline Characteristic & $\begin{array}{l}\text { Pre-COVID } \\
\text { Cohort } \\
\mathbf{n}(\%)\end{array}$ & $\begin{array}{l}\text { During COVID } \\
\text { Cohort } \\
\mathbf{n}(\%)\end{array}$ & p-value \\
\hline Prophylactic G-tube & $8(5 \%)$ & $11(8 \%)$ & 0.30 \\
\hline Reactive G-tube & $17(11 \%)$ & $20(15 \%)$ & 0.32 \\
\hline Nasogastric tube & $11(7 \%)$ & $10(7 \%)$ & 0.91 \\
\hline Any enteral feeding & $33(21 \%)$ & $41(30 \%)$ & 0.07 \\
\hline Mean percentage weight loss & $-5.6 \%$ & $-6.8 \%$ & 0.03 \\
\hline Greater than 10\% weight loss & $34(22 \%)$ & $42(31 \%)$ & 0.07 \\
\hline Aspiration pneumonia & $10(6 \%)$ & $4(3 \%)$ & 0.17 \\
\hline Hospitalization & $24(15 \%)$ & $21(15 \%)$ & 0.97 \\
\hline
\end{tabular}


Table 3

Mean average scores for each symptom question / subscale total across the 7 weeks of radiation treatment.

\begin{tabular}{|c|c|c|c|c|c|c|}
\hline \multirow[t]{3}{*}{ Question } & \multirow[t]{3}{*}{ Symptom } & \multicolumn{4}{|c|}{ Average Score } & \multirow[t]{3}{*}{ p-value } \\
\hline & & \multicolumn{2}{|c|}{ Pre-COVID Cohort } & \multicolumn{2}{|c|}{ During COVID Cohort } & \\
\hline & & Mean & SD & Mean & SD & \\
\hline 1 & Pain & 3.75 & 2.29 & 4.05 & 2.32 & 0.27 \\
\hline 2 & Fatigue/tiredness & 4.15 & 2.23 & 4.54 & 2.31 & 0.16 \\
\hline 3 & Nausea & 2.23 & 2.32 & 2.59 & 2.30 & 0.19 \\
\hline 4 & Disturbed sleep & 3.41 & 2.35 & 3.60 & 2.40 & 0.49 \\
\hline 5 & Distressed/upset & 2.63 & 2.17 & 3.03 & 2.42 & 0.15 \\
\hline 6 & Shortness of breath & 1.65 & 1.98 & 1.72 & 2.09 & 0.77 \\
\hline 7 & Remembering things & 2.00 & 1.96 & 2.21 & 1.95 & 0.36 \\
\hline 8 & Lack of appetite & 3.81 & 2.69 & 4.38 & 2.46 & 0.068 \\
\hline 9 & Drowsy/sleepy & 3.48 & 2.26 & 3.95 & 2.31 & 0.090 \\
\hline 10 & Dry mouth & 4.39 & 0.21 & 4.55 & 0.22 & 0.60 \\
\hline 11 & Feeling sad & 2.19 & 2.25 & 2.71 & 2.48 & 0.072 \\
\hline 12 & Vomiting & 0.88 & 1.55 & 1.40 & 1.98 & 0.017 \\
\hline 13 & Numbness/tingling & 1.29 & 1.80 & 1.45 & 1.78 & 0.47 \\
\hline 14 & Mucus & 3.62 & 2.44 & 4.02 & 2.60 & 0.19 \\
\hline 15 & swallowing/chewing & 4.08 & 2.44 & 4.58 & 2.42 & 0.092 \\
\hline 16 & Choking/coughing & 1.83 & 1.97 & 2.49 & 2.01 & 0.0065 \\
\hline 17 & Voice/speech & 3.00 & 2.50 & 3.13 & 2.42 & 0.68 \\
\hline 18 & Skin pain/burning/rash & 2.41 & 2.07 & 2.81 & 2.06 & 0.11 \\
\hline 19 & Constipation & 2.65 & 2.31 & 2.83 & 2.42 & 0.54 \\
\hline 20 & Tasting food & 4.84 & 3.19 & 5.31 & 2.66 & 0.18 \\
\hline 21 & Mouth/throat sores & 3.14 & 2.45 & 3.73 & 2.39 & 0.04 \\
\hline 22 & Teeth/gums & 2.09 & 2.17 & 2.59 & 2.25 & 0.06 \\
\hline
\end{tabular}

Symptom severity differences between cohorts were compared using independent t-tests with Bonferroni correction. Group differences were considered significant if $p<0.002$ for the 28 individual symptom items and $p<0.02$ for the 3 subscale totals. 


\begin{tabular}{|c|c|c|c|c|c|c|}
\hline \multirow[t]{3}{*}{ Question } & \multirow[t]{3}{*}{ Symptom } & \multicolumn{4}{|c|}{ Average Score } & \multirow[t]{3}{*}{ p-value } \\
\hline & & \multicolumn{2}{|c|}{ Pre-COVID Cohort } & \multicolumn{2}{|c|}{ During COVID Cohort } & \\
\hline & & Mean & SD & Mean & SD & \\
\hline & Interference & & & & & \\
\hline 23 & General activity & 3.40 & 2.41 & 3.61 & 2.57 & 0.48 \\
\hline 24 & Mood & 2.81 & 2.27 & 3.27 & 2.48 & 0.11 \\
\hline 25 & Work/housework & 3.54 & 2.65 & 3.65 & 2.58 & 0.71 \\
\hline 26 & Relations & 2.35 & 2.25 & 2.55 & 2.31 & 0.47 \\
\hline 27 & Walking & 2.49 & 2.28 & 2.54 & 2.37 & 0.87 \\
\hline \multirow[t]{2}{*}{28} & Enjoyment of life & 3.48 & 2.52 & 3.76 & 2.73 & 0.38 \\
\hline & Subscale Totals & & & & & \\
\hline $1-13$ & Core Symptoms & 2.74 & 1.62 & 3.08 & 1.73 & 0.093 \\
\hline $14-22$ & H\&N Module Symptoms & 3.05 & 1.74 & 3.49 & 1.82 & 0.042 \\
\hline $23-28$ & Interference & 2.99 & 2.17 & 3.18 & 2.30 & 0.46 \\
\hline $\begin{array}{l}\text { Symptom } \\
\text { Bonferror } \\
\text { symptom }\end{array}$ & $\begin{array}{l}\text { verity differences between } \\
\text { rrection. Group difference } \\
\text { ms and } p<0.02 \text { for the } 3 \mathrm{~s}\end{array}$ & $\begin{array}{l}\text { orts we } \\
\text { ere cons } \\
\text { cale tot }\end{array}$ & $\begin{array}{l}\text { mparec } \\
\text { ed signi }\end{array}$ & $\begin{array}{l}\text { sing ind } \\
\text { ant if } p\end{array}$ & $\begin{array}{l}\text { nt t-tests } \\
\text { for the } 2\end{array}$ & dividual \\
\hline
\end{tabular}

\section{Univariate Linear Mixed Model Analysis}

The results of the mixed model analyses indicates that across all symptom scores, within subject tests reveal a significant time effect: symptom severity became significantly worse over the duration of radiotherapy (all $p<0.0001$ ). Select between cohort symptom scores are shown in Fig. 1 . There were three symptom scores with a significant cohort effect detected, with higher symptom scores during COVID versus pre COVID. These included vomiting $(p=0.01)$, choking/coughing while eating $(p=0.007)$ and mouth/throat sores $(p=0.04)$. Figure 2 shows the mean symptom scores for the Core, $H \& N$ and Interference subscales during COVID versus pre COVID. The scores were significantly higher for the H\&N subscale during COVID versus pre COVID $(p=0.04)$.

\section{Multivariate Linear Mixed Model Analysis}

Results of multivariate mixed model analyses indicate that symptom severity becomes significantly worse over time for all symptoms evaluated (all $p<0.0001$ ).

I. Q12 - Vomiting 
Symptoms of vomiting became significantly worse over time for all patients $(p<0.0001)$. Node positivity was associated with higher mean symptom severity $(1.7$ vs. $1.1, p=0.026)$. Paranasal sinus/nasopharynx tumor sites (vs salivary gland) were associated with higher mean symptoms of vomiting ( 2.1 vs. $0.8, p=0.016)$. Non-smokers $(1.2$ vs. $1.9 p=0.033)$ and ex-smokers $(1.3$ vs.1.9 $p=0.046)$ had less severe symptoms of vomiting compared to current smokers, and patients who experienced less weight loss reported lower vomiting scores $(p=0.0006)$. Treatment pre versus during the COVID pandemic was not associated with vomiting score on multivariable analysis $(p=0.12)$.

II. Q16 - Choking/coughing while eating

Symptoms of choking/coughing while eating became significantly worse over time for all patients $(\mathrm{p}<$ 0.0001) Treatment during COVID compared to pre COVID was associated with higher choking/coughing scores (2.7 vs. $2.1, p=0.013)$. Higher mean scores were reported for female patients ( 2.8 vs. $2, p=0.01)$ and those with node positive disease ( 2.8 vs. $2, p=0.01)$, whereas patients with reduced weight loss $(p=$ $0.008)$ and higher doses of RT $(p=0.032)$ had less severe symptoms of choking/coughing.

III. Q21 - Mouth/throat sores

Symptoms of mouth/throat sores became significantly worse over time for all patients $(p<0.0001)$. Node positivity was associated with greater mean severity of mouth sores $(4.4$ vs. $2.9, p<0.0001)$. The cohort*time interaction remained significant in the multivariate model for mouth/throat sores $(p=0.019)$ indicating the severity of the symptom may be experienced differently for patients treated during COVID compared to pre COVID (i.e., although mouth sores are becoming worse over time for all patients, the severity is changing at different rates for the two cohorts). Treatment pre versus during the COVID pandemic was not associated with mouth/throat sore severity on multivariable analysis $(p=0.09)$.

\section{Core symptom subscale}

Overall core symptoms became significantly worse over time for all patients $(p<0.0001)$. Node positivity was associated with higher mean core symptoms (3.7 vs. 2.9, $p=0.001)$. Paranasal sinus/nasopharynx tumor sites (vs salivary gland) was associated with higher mean core symptom scores $(p=0.012)$. Patients who experienced less weight loss $(p=0.002)$ and had better performance status (ECOG $0 / 1$ vs $2 / 3 ; 2.9$ vs. $3.7 p=0.036$ ) reported lower mean core symptoms. Treatment pre versus during the COVID pandemic was not associated with core symptom severity on multivariable analysis $(p=0.27)$.

\section{H\&N symptom subscales}

$\mathrm{H} \& \mathrm{~N}$ symptoms became significantly worse over time for all patients $(\mathrm{p}<0.0001)$. Node positivity was associated with symptom severity (4.2 vs. 3.2, $p=0.0001)$. Paranasal sinus/nasopharynx tumor sites (vs Salivary gland) was associated with more severe H\&N symptoms ( 4.2 vs. $3, p=0.026)$. Patients who experienced less weight loss $(p=0.0001)$ and had better ECOG (ECOG 0/1 vs $2 / 3 ; 3.2$ vs. $4.1, p=0.015)$ reported lower mean H\&N symptoms. Treatment pre versus during the COVID pandemic was not associated with H\&N subscale symptom severity on multivariable analysis $(p=0.12)$. 
Overall symptoms of interference became significantly worse over time for all patients $(p<0.0001)$. Node positivity was associated with higher mean symptom severity (4.2 vs. 3.1, p=0.001). Paranasal sinus/nasopharynx tumor sites (vs salivary gland) was significantly associated with higher mean interference symptoms (4.7 vs. 2.7, $\mathrm{p}=0.003)$. Patients with better ECOG performance (ECOG 0/1 vs 2/3) have lower mean interference symptom scores (3 vs. 4.3, $p=0.008$ ) Treatment pre versus during the COVID pandemic was not associated with interference severity on multivariable analysis $(p=0.68)$.

\section{Discussion:}

This study is the first, to our knowledge, evaluating patient reported outcomes during radical radiotherapy for H\&N cancer, comparing patients treated pre-COVID-19 pandemic versus during the COVID-19 pandemic. The two cohorts were very similar at baseline with respect to demographics, clinical variables and treatment variables, although there were more smokers and usage of chemotherapy in the COVID-19 era. Patients reported more severe choking and coughing while eating during the COVID-19 pandemic and had a greater percentage weight loss from baseline. There were no significant differences between treatment cohorts for any of the other symptom outcomes examined in multivariate analysis.

During the COVID-19 pandemic, several organizations suggested possible mitigation strategies including using concurrent chemoradiotherapy if there are prolonged operating room closures and altering radiation-dose fractionation schedules to a shorter hypofractionated course.[7], [12], [13] At our institution, we did not change our standard of care treatment recommendations during the COVID-19 pandemic. In the ASTRO-ESTRO guidelines published by Thomson et al. there was strong agreement to modify the weekly in-person on-treatment reviews for H\&N patients during radiotherapy.[12] One quarter of respondents (23\%) had stopped in-person reviews completely and others had reduced the weekly frequency of in-person appointments, replacing them with telephone (50\%) or video (26\%) reviews instead.[12][14] At our institution, we continued in person radiation oncology visits for all weekly on treatment visits for $\mathrm{H} \& \mathrm{~N}$ cancer patients, as they were already present in the radiotherapy department, unless the patient requested a virtual appointment, which was very uncommon. It is possible this contributed to the similar PROs as pre-pandemic, as symptoms were continued to be evaluated and managed in person with accompanying physical examination by radiation oncologists weekly. A proportion of medical oncology, dietician and speech language pathology appointments were performed virtually during the pandemic.

The incidence of complications during radiotherapy such as unplanned hospitalization, aspiration pneumonia, and enteral feeding requirements were similar during the pandemic versus pre-pandemic. However, patients did have a greater mean percentage weight loss relative to their baseline during the COVID-19 pandemic. During the pandemic, patients continued to have a weekly consultation with a registered dietician. These appointments were a mix of in-person versus telephone appointments. The difference in mean weight loss between the cohorts was only $1.2 \%$, and this may not be considered a 
clinically relevant finding given the small absolute difference. A potential explanation is that during the pandemic, many patients had less in-person support from their family and friends due to social distancing regulations that were in place. A previous study showed that greater than $10 \%$ weight loss was associated with lower social contact scores.[15] None of the patients in our cohort developed COVID-19 infection and prior studies have shown a mortality rate of $18.9 \%$ in head and neck cancer patients on active treatment.[16]

On both univariate and multivariate analysis, receiving radiation during the COVID-19 pandemic was associated with higher patient reported choking/coughing while eating relative to pre-pandemic. Before the pandemic, all patients at our institution had a pre-treatment in-person speech language pathology (SLP) consultation with a bedside swallowing assessment. Patients were then seen in person, weekly or biweekly during radiotherapy, and instrumental assessments (VFSS and FEES) were performed as appropriate. During the pandemic, patients were seem via Zoom or telephone pre-treatment and weekly or biweekly during treatment. It is possible that the sensitivity of detecting worsening dysphagia via verbal communication alone was reduced. This may also be more likely in a population such as $\mathrm{H} \& \mathrm{~N}$ patients who may be experiencing difficulties with speech due to mucositis, hoarseness and pain. Obtaining VFSS and FEES instrumental assessments were also greatly limited during the pandemic, based on published recommendations, due to the risk of COVID transmission during aerosol generating medical procedures and limited fluoroscopic room availabilities.[17] Finally, pre-pandemic, the multidisciplinary group met weekly to discuss challenging cases, which alerted the SLP team earlier to patients struggling with new or worsening dysphagia. During the pandemic, multidisciplinary group meetings were not scheduled during the study era; however, were later established in a virtual format to provide better team based care.[18]

To date, there have been very few published studies evaluating the quality of life of head and neck cancer patients during the COVID-19 pandemic. A survey by Gallo et al. of $121 \mathrm{H} \& \mathrm{~N}$ cancer patients were compared to a historical cohort.[19] They found that during the pandemic, H\&N cancer survivors reported worse social functioning, physical functioning and dyspnea relative to a similarly matched historical cohort. We did not see these differences in our cohort, based on the shortness of breath symptom assessment and Interference subscales, however, Gallo et al. used the EORTC QLQ-C30 measure, which cannot be directly compared to MDASI-HN. Additionally, this population of patients was post-treatment, whereas our population included only those on active radiotherapy.[19] A large study of cancer patients with a variety of tumour subsites, including 77 head and neck cancer patients, reported the majority of patients were very concerned about the impact of COVID-19 on their cancer treatment, highlighting the importance of psychosocial support needed during the pandemic.[20]

Several studies have shown a delay in diagnosis and treatment of head and neck cancer during the pandemic. [6], [7], [21], [22] [23]There are several potential explanations for this, including fear of patients to attend hospitals or physicians offices, reduced in-person consultations and specialist availability, and reduced operating room availability and post anesthesia care for complex head and neck cancer surgeries. [6], [7], [21], [22] Increased time to surgery in head and neck cancer is associated with higher morbidity and mortality.[22] It is possible that the patients in our study treated during the COVID-19 
pandemic experienced delays in diagnosis. However, the stage and tumour site distributions and ECOG status were similar in both cohorts, and delays in diagnosis is beyond the evaluation and objectivesof this particular study.

Our study has both strengths and limitations. To our knowledge, this is the first study comprehensively comparing patient reported outcomes and complications between a large cohort of head and neck cancer patients undergoing radiotherapy pre COVID-19 relative to during COVID 19 and comprehensively following the trajectory of symptoms during radiotherapy. Limitations to our study include that given the variability of COVID-19 incidence and impact across the globe and subsequent impact on oncologic care (operating room time, availability of in-person assessments and provision of multi-disciplinary care), our results may not have external validity to other institutions. Additionally, we did not evaluate the impact of potential delay in diagnosis during the COVID-19 pandemic and subsequent influence on patient reported outcomes, as this was outside the focus of this study.

In conclusion, head and neck cancer patients at our institution during the COVID-19 pandemic were similar at baseline relative to patients treated pre-COVID-19 pandemic. During the pandemic, on-treatment reviews were continued by radiation oncologists in person, but many support services were shifted to telehealth to maintain social distancing recommendations. Patients reported more severe choking and coughing while eating during the COVID-19 pandemic and had a greater percentage weight loss from baseline, but the remainder of patient reported outcomes and complications during radiotherapy were similar between the two cohorts.

\section{Declarations}

Funding: This work was supported by Varian Medical Systems through an unrestricted grant.

Conflicts of interest/Competing interests: $\mathrm{SH}, \mathrm{ET}, \mathrm{JW}, \mathrm{KD}, \mathrm{VL}, \mathrm{MC}, \mathrm{MC}$ report no disclosures. $\mathrm{RO}$ has received grants from Varian Medical Systems related to this work. EB has received grants and personal fees from Genzyme and Eisai outside the submitted work NC reports honoraria from Bayer, Eisai, Merck, and Roche Canada; has served as a consultant or advisor to Bayer, Eisai, Lilly, Merck, and Roche; has received research funding from GlaxoSmithKline; and has received institutional research funding from Merck and Pfizer all outside the submitted work.

Availability of data and material: Data will not be shared due to privacy restrictions at our institution.

Code availability: Not applicable

Authors' contributions: All contributed to this manuscript and reviewed the final version.

Ethics approval: UBC Research Ethics Board approved.

Consent to participate: Not applicable 


\section{References}

1. Niska JR, Halyard MY, Tan AD, Atherton PJ, Patel SH, Sloan JA, "Electronic patient-reported outcomes and toxicities during radiotherapy for head-and-neck cancer," Qual. Life Res., vol. 26, no. 7, pp. 17211731, Jul. 2017, doi: 10.1007/s11136-017-1528-2

2. Falchook $A D$ et al., "Comparison of patient- and practitioner-reported toxic effects associated with chemoradiotherapy for head and neck cancer," JAMA Otolaryngol. - Head Neck Surg., vol. 142, no. 6, pp. 517-523, Jun. 2016, doi: 10.1001/jamaoto.2016.0656

3. Pusic A et al (2007) A systematic review of patient-reported outcome measures in head and neck cancer surgery. Otolaryngol - Head Neck Surg 136(4):525-535. doi:10.1016/j.otohns.2006.12.006

4. Wilkie JR et al., "Big data analysis of associations between patient reported outcomes, observer reported toxicities, and overall quality of life in head and neck cancer patients treated with radiation therapy," Radiother. Oncol., vol. 137, pp. 167-174, Aug. 2019, doi: 10.1016/j.radonc.2019.04.030

5. Ringash J, "Survivorship and quality of life in head and neck cancer," Journal of Clinical Oncology, vol. 33, no. 29. American Society of Clinical Oncology, pp. 3322-3327, 10-Oct-2015, doi:

$10.1200 / J C 0.2015 .61 .4115$

6. Tevetoğlu F, Kara S, Aliyeva C, Yıldııım R, Yener HM (2021) Delayed presentation of head and neck cancer patients during COVID-19 pandemic. Eur Arch Oto-Rhino-Laryngology. doi:10.1007/s00405021-06728-2

7. Brody RM et al (2020) Changes in head and neck oncologic practice during the COVID-19 pandemic. in Head Neck 42(7):1448-1453. doi:10.1002/hed.26233

8. Olson RA et al., "Provincial development of a patient-reported outcome initiative to guide patient care, quality improvement, and research," Healthc. Manag. Forum, vol. 31, no. 1, Jan. 2018, doi:

$10.1177 / 0840470417715478$

9. Holländer-Mieritz C, Johansen J, Johansen C, Vogelius IR, Kristensen CA, Pappot H (May 2019) Comparing the patients' subjective experiences of acute side effects during radiotherapy for head and neck cancer with four different patient-reported outcomes questionnaires. Acta Oncol (Madr) 58(5):603-609. doi:10.1080/0284186X.2018.1563713

10. Rosenthal DI et al (Jul. 2014) Patterns of symptom burden during radiotherapy or concurrent chemoradiotherapy for head and neck cancer: A prospective analysis using the University of Texas MD Anderson Cancer Center Symptom Inventory-Head and Neck Module. Cancer 120(13):19751984. doi:10.1002/cncr.28672

11. Cleeland CS, “The MD Anderson Symptom Inventory User Guide • Version 1," 2016

12. Thomson DJ et al., "Practice Recommendations for Risk-Adapted Head and Neck Cancer Radiation Therapy During the COVID-19 Pandemic: An ASTRO-ESTRO Consensus Statement," Int. J. Radiat. Oncol. Biol. Phys., vol. 107, no. 4, pp. 618-627, Jul. 2020, doi: 10.1016/j.jjrobp.2020.04.016 
13. Sharma A, Crosby DL (2020) Special considerations for elderly patients with head and neck cancer during the COVID-19 pandemic. in Head Neck 42(6):1147-1149. doi:10.1002/hed.26216

14. Prasad A, Brewster R, Newman JG, Rajasekaran K, "Optimizing your telemedicine visit during the COVID-19 pandemic: Practice guidelines for patients with head and neck cancer," in Head and Neck, 2020, vol. 42, no. 6, pp. 1317-1321, doi: 10.1002/hed.26197

15. Langius JAE et al (2013) More than $10 \%$ weight loss in head and neck cancer patients during radiotherapy is independently associated with deterioration in quality of life. Nutr Cancer 65(1):7683. doi:10.1080/01635581.2013.741749

16. Hanna GJ et al., "Hospitalization rates and 30-day all-cause mortality among head and neck cancer patients and survivors with COVID-19," Oral Oncol, 112, Jan. 2021, doi:10.1016/j.oraloncology.2020.105087

17. Ku PKM et al (2020) Management of dysphagia in the patient with head and neck cancer during COVID-19 pandemic: Practical strategy. in Head Neck 42(7):1491-1496. doi:10.1002/hed.26224

18. Nilsen ML et al (2020) Prevision of multidisciplinary head and neck cancer survivorship care during the 2019 novel coronavirus pandemic. in Head Neck 42(7):1668-1673. doi:10.1002/hed.26256

19. Gallo 0 et al (2021) The impact of the COVID-19 pandemic on the quality of life of head and neck cancer survivors. Support Care Cancer. doi:10.1007/s00520-021-06198-6

20. de Joode $\mathrm{K}$ et al (Sep. 2020) Impact of the coronavirus disease 2019 pandemic on cancer treatment: the patients' perspective. Eur J Cancer 136:132-139. doi:10.1016/j.ejca.2020.06.019

21. Shaw R et al., "Head and neck cancer surgery during the COVID-19 pandemic: An international, multicenter, observational cohort study," Cancer, vol. 127, no. 14, pp. 2476-2488, Jul. 2021, doi: 10.1002/cncr.33320

22. Rygalski CJ et al (Feb. 2021) Time to Surgery and Survival in Head and Neck Cancer. Ann Surg Oncol 28(2):877-885. doi:10.1245/s10434-020-09326-4

23. Chaves ALF et al., "Emergency changes in international guidelines on treatment for head and neck cancer patients during the COVID-19 pandemic," Oral Oncology, vol. 107. Elsevier Ltd, 01-Aug-2020, doi: 10.1016/j.oraloncology.2020.104734

\section{Figures}



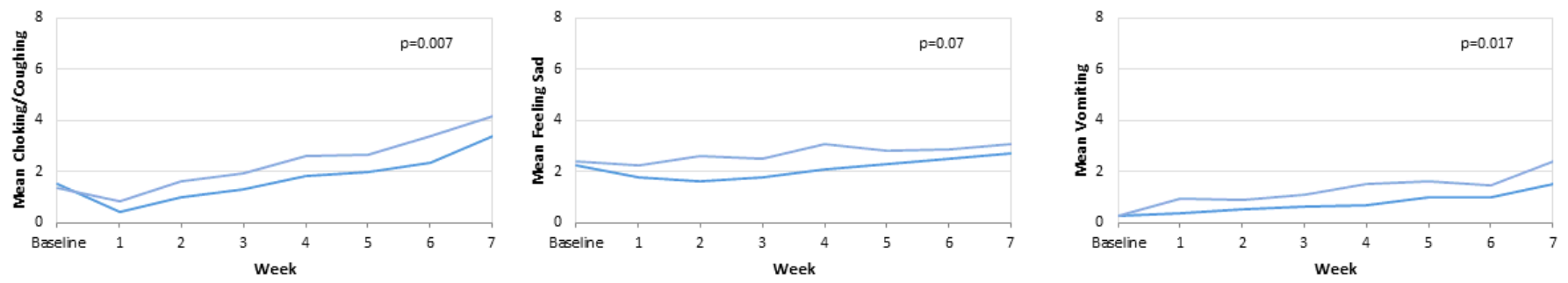

- Pre COVID —During COVID

- Pre COVID During COVID
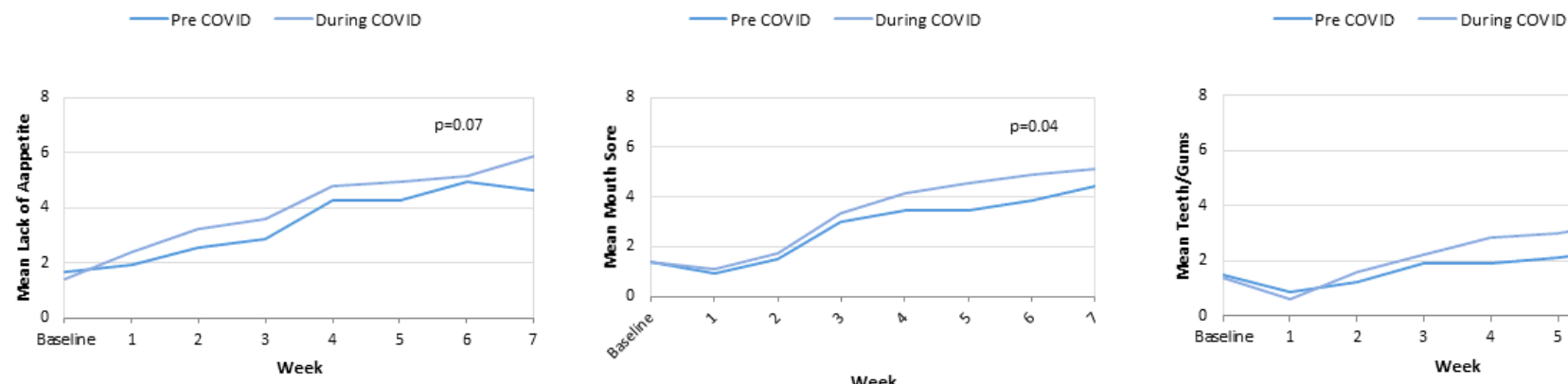

- Pre COVID D During COVID
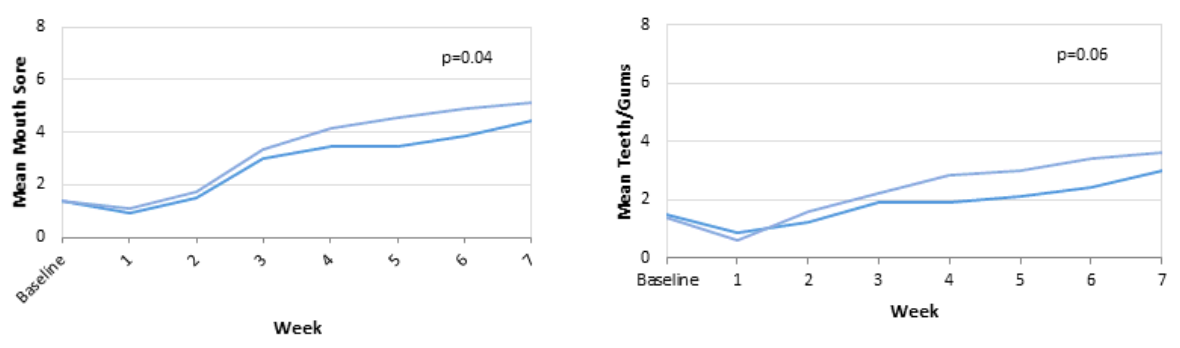

- Pre COVID During COVID

- Pre COVID During COVID

Figure 1

Mean symptom scores pre-COVID vs during COVID for select individual symptoms by week of radiotherapy.

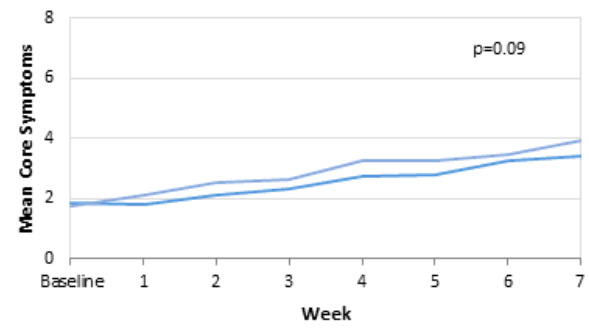

- Pre COVID D During COVID

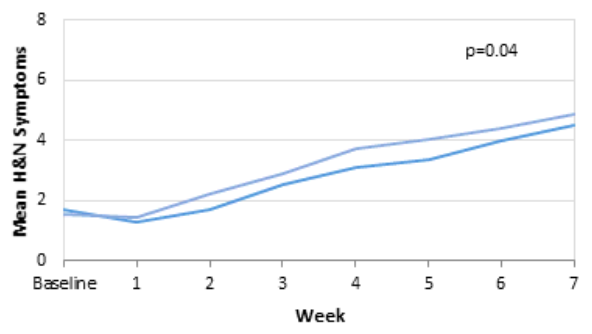

- Pre COVID During COVID

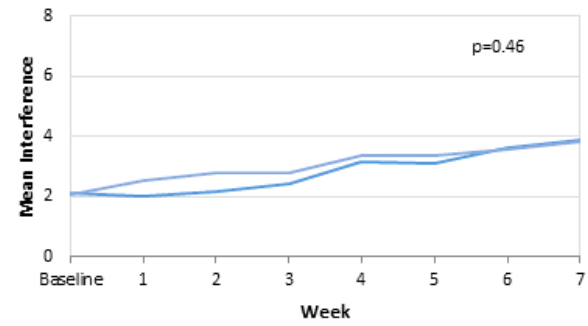

- Pre COVID During COVID

\section{Figure 2}

Mean symptom scores pre-COVID vs during COVID for Core Symptoms, H\&N Symptoms, and Interference by week of radiotherapy 